\title{
IL-19 Induced by IL-13/IL-17A in the Nasal Epithelium of Patients with Chronic Rhinosinusitis Upregulates MMP-9 Expression via ERK/NF-kB Signaling Pathway
}

\section{Xia Li}

Third Affiliated Hospital of Sun Yat-Sen University

Jiancong Huang

Third Affiliated Hospital of Sun Yat-Sen University

\section{Xiaohong Chen}

Third Affiliated Hospital of Sun Yat-Sen University

\section{Xiaoping Lai}

Third Affiliated Hospital of Sun Yat-Sen University

\section{Zizhen Huang}

Third Affiliated Hospital of Sun Yat-Sen University

\section{Yue Li}

Third Affiliated Hospital of Sun Yat-Sen University

\section{Shuaixiang Li}

Third Affiliated Hospital of Sun Yat-Sen University

\section{Lihong Chang}

Third Affiliated Hospital of Sun Yat-Sen University

Gehua Zhang ( $\sim$ zhanggeh@mail.sysu.edu.cn )

Third Affiliated Hospital of Sun Yat-Sen University https://orcid.org/0000-0002-1917-0283

\section{Research}

Keywords: chronic rhinosinusitis, IL-19, MMP-9, nasal epithelium

Posted Date: July 28th, 2020

DOI: https://doi.org/10.21203/rs.3.rs-46150/v1

License: (c) (i) This work is licensed under a Creative Commons Attribution 4.0 International License. Read Full License 


\section{Abstract \\ Background}

Tissue remodeling is a crucial characteristic of chronic rhinosinusitis (CRS). Imbalance between matrix metalloproteinases (MMPs) and tissue inhibitors of metalloproteinases (TIMPs) is crucial for the pathologic tissue remodeling in CRS. Elevation of interleukin (IL)-19 or MMP-9 levels in patients with CRS had been proven in previous studies. Here, we aimed to investigate the role of IL-19 in mediating MMP-9 expression in CRS.

\section{Methods}

Nasal tissue samples were collected from individuals having chronic rhinosinusitis with nasal polyps (CRSwNP), CRS without nasal polyps (CRSsNP), and controls. Expression of IL-19, its receptors (IL20R1/IL-20R2), and MMP-9 were investigated. Human nasal epithelial cells (HNECs) were stimulated by IL-19; ERK phosphorylation, NF-KB pathway activation, and MMP-9 level were detected. We also explored the effect of type1/2/3 cytokines on IL-19 production.

\section{Results}

Expression levels of IL-19, its receptors (IL-20R1/IL-20R2), and MMP-9 were increased in nasal tissues from individuals with CRSwNP compared to those with CRSsNP as well as the controls. IL-19 significantly elevated the production of MMP-9 in HNECs. Furthermore, IL-19 could activate the ERK and NF-KB pathways, accompanied by increased MMP-9 production in HNECs. Conversely, both ERK and NF$\mathrm{KB}$ inhibitors significantly attenuated the role of IL-19 in MMP-9 production. siRNA knockdown of IL-20R1 suppressed ERK and NF-KB pathway activation, thereby decreasing MMP-9 expression. IL-13 and IL-17A were found to stimulate IL-19 production in HNECs.

\section{Conclusion}

IL-19, promoted by IL-13 and IL-17A, contributes to the upregulation of secretion of the tissue remodeling factor MMP-9 in patients with CRS.

\section{1 | Introduction}

Chronic sinusitis (CRS) is a common disease, with prevalence of $8 \%$ in China and $12 \%$ in the United States.[1-3] It is a considerable public health concern as well as a socioeconomic burden.[4] Clinically, CRS is categorized into two types: CRS with (CRSwNP) or without nasal polyps (CRSsNP). Histologically, CRSsNP is predominantly characterized by basement membrane thickening and interstitial fibrosis. On the contrary, CRSwNP is characterized by stromal tissue edema with albumin deposition and pseudocyst 
formation.[5] Moreover, CRSwNP is considered a heterogeneous disease, and based on the expression of different inflammatory cytokines in CRSwNP, it can be divided into different clusters. Clinical characteristics, such as treatment outcomes, asthma prevalence, and postoperative recurrence, are significantly different across the clusters.[6, 7] Despite the variation across subtypes of CRSwNP, manifestation of tissue remodeling is similar, hence indicating different inflammatory cytokines to eventually lead to similar tissue remodeling pattern through common downstream factors or pathways in nasal polyps.

Tissue remodeling in CRS involves transformation of the tissue structure and extracellular matrix (ECM). Several elements involved in tissue remodeling include MMPs, TIMPs, and transforming growth factor (TGF)- $\beta$. Imbalance between MMPs and TIMPs is considered to be the key factor for pathological tissue remodeling in CRS.[8, 9] As a family of zinc-dependent proteolytic enzyme, MMPs can degrade a variety of constituents of ECM and regulate remodeling.[10] However, MMP-1, MMP-2, and MMP-3 expression in patients with CRS is controversial, whereas several studies have reported MMP-7 and MMP-9 levels to be higher in cases with CRSWNP.[11-14] Overall, the detailed upstream modulation of MMPs promoting tissue remodeling in chronic sinusitis remains unclear.

IL-19 is one of the members of IL-20 cytokine subfamily, which also includes IL-20, IL-22, IL-24, and IL-26. [15] The structures of IL-20 subfamily receptor heterodimers are somewhat similar. IL-19 needs to bind to a functional heterodimeric receptor IL-20R1/IL-20R2 for mediating its signal transduction.[16] Studies concerning IL-20 subfamily have shown IL-20 to induce MMP-3 in primarily cultured human disc cells[17]; IL-20 had also been reported to significantly promote MMP-9 production in bladder cancer cells. [18] E. Pace et.al had found IL-19 to be overexpressed in the epithelium of patients with CRSwNP [19]; however, whether IL-19 mediates MMP-9 expression in CRS, and if so, by what mechanism, still remains unclear.

Recent studies have suggested tissue remodeling of CRS to occur concurrently with inflammation.[20] Studies, principally in Chinese patients, have shown CRSsNP to be characterized by type 1 inflammatory patterns while CRSwNP was characterized by mixed type $2 / 3$ inflammatory profiles.[21, 22] Whether IL-19 could serve as a common downstream factor of type $1 / 2 / 3$ cytokines in nasal epithelia, resulting in the regulation of MMP-9, remains unknown. This study aimed to confirm the role of IL-19 in regulating MMPs expression and explore the effect of type 1/2/3 cytokines on IL-19 production in Human nasal epithelial cells (HNECs) of patients with CRS.

\section{2 | Materials And Methods}

\section{1 | Patients and tissue samples}

We diagnosed and classified CRS according to the EPOS 2012 guidelines.[23] Polyps from patients diagnosed with CRSwNP were obtained along with uncinate process and sinus mucosal tissues from patients with CRSsNP; control samples were collected from patients without sinonasal inflammation. A part of the samples was stored at $-80^{\circ} \mathrm{C}$, and subsequently used for studying histologic changes, RNA 
and protein isolation, and immunofluorescence. The rest was freshly submerged in PBS supplemented with $1 \%$ antibiotic-antimycotic for subsequent HNECs culture. The present study was approved by the ethical committee of the Third Affiliated Hospital of Sun Yat-Sen University. All subjects were enrolled in the study only after acquiring informed consent from them. More details can be found in the supplementary material.

\section{2 | Human nasal epithelial cells culture}

Human nasal tissues were cut into $1 \times 1-\mathrm{mm}$ fragments, digested in Dispase II (50 mg/mL, Sigma-Aldrich, St. Louis, MO, USA) overnight at $4^{\circ} \mathrm{C}$, followed by further digestion in trypsin for $15 \mathrm{~min}$ at $37^{\circ} \mathrm{C}$; the digestion was terminated by adding complete DMEM containing $10 \%$ FBS. The digested nasal tissue pieces were then filtered by a 100-mm cell strainer to obtained cells. Cells were then suspended in complete DMEM and cultured in an incubator for $30 \mathrm{~min}$ to remove fibroblasts. The obtained HNECs were then cultured in BEGM (Lonza, Basel, Switzerland).

\section{3 | Real-time quantitative PCR (RT-qPCR)}

RT-qPCR was performed, as described previously, to detect the mRNA level of target genes.[24] More details can be found in online supplementary material.

\section{4 | ELISA}

Supernatant of HNECs was collected and examined by MMP-9 ELISA kits (CUSABIO, Wuhan, China). All procedures followed the manufacturers' instructions.

\section{5 | Western blot}

Western blotting was performed as reported previously.[13] The PVDF membranes were first incubated with primary antibodies MMP-9 (1:1000; Abcam, Cambridge, UK), pERK, ERK, plкBa, ІкBa (1:1000; Cell Signaling Technology, Boston, USA), and GAPDH (1:3000; PeproTech, Rocky Hill, USA) overnight at $4{ }^{\circ} \mathrm{C}$ separately, and then incubated with HRP-conjugated secondary antibody (Bioworld, Nanjing, China). Color development was achieved by ECL reagents. More information can be found in the online supplementary material.

\section{6 | Immunofluorescence(IF) staining and confocal microscopy}

The frozen sections from human nasal tissue were blocked with $10 \%$ goat serum for 30 min and stained first with IL-19 (1:50; Abcam, Cambridge, UK) and MMP-9 antibodies (1:50; Abcam) overnight at $4{ }^{\circ} \mathrm{C}$, separately. HNECs were fixed with $4 \%$ paraformaldehyde, blocked in 1\% BSA for 30 min, and then stained first with MMP-9 antibodies or p65 (1:500; Cell Signaling Technology, Boston, USA) overnight at $4{ }^{\circ} \mathrm{C}$. Subsequently, the frozen tissue sections and HNECs were incubated with Alexa Fluor 594-/or 488conjugated secondary antibodies (1:1000; Invitrogen, California, USA) for $1 \mathrm{~h}$ at room temperature. Finally, the slices were stained with DAPI (1:30000; Biolegend, California, USA) to mark the nuclei, and 
sealed with antifade mountant (Invitrogen, California, USA). Immunofluorescence was examined at 400x magnification under a microscope (Leica, Wetzlar, Germany), or at 630x magnification in a confocal microscope (Carl Zeiss, Jena, Germany).

\section{7 | siRNA transfection and ERK and NF-kB pathway inhibition}

Human IL-20R1 siRNA (5'-CUUACACUGUGCAGUAUUUUU-3') was synthesized by IGE Biotechnology company (Guangzhou, China). For siRNA transfection, HNECs were transfected for $48 \mathrm{~h}$ using Lipofectamine $^{\text {TM }} 3000$ Reagent (Invitrogen, California, USA), in line with the recommended protocol. Subsequently, HNECs were stimulated with $100 \mathrm{ng} / \mathrm{mL}$ IL-19 (or without) for $24 \mathrm{~h}$. For pathway inhibition, ERK and NF-kB pathways were suppressed by inhibitors PD98059 or BAY 11-7082 (MedChem Express, Monmouth Junction, NJ, USA) for $1 \mathrm{~h}$, respectively. Similarly, HNECs were stimulated with IL-19 for $24 \mathrm{~h}$.

\section{8 | Statistical analysis}

IBM SPSS 20 (SPSS, Chicago, IL, USA) was used for statistical analyses. When data were normally distributed, they were presented as mean \pm SEM; otherwise, they were presented as median (25-75 percentiles). One-way ANOVA or Kruskal-Wallis test was applied to analyze significance across groups, for comparative study. Student's $t$ or Mann-Whitney U test (2-tailed) was then applied for comparisons across groups. Paired Student's $t$ or Wilcoxon matched-pair signed rank test was used when suitable. $P<0.05$ was regarded as significant.

\section{3 | Results}

\subsection{Increased expression of IL-19, its receptors (IL-20R1/IL-20R2), and MMP-9 in nasal mucosa of patients with CRSwNP}

Tissues collected from patients with CRSWNP and CRSsNP, and from controls, were examined for IL-19, its receptors, and MMP-9 expression by RT-qPCR. Results demonstrated IL-19, its receptors (IL-20R1/IL20R2), and MMP-9 mRNA expression to be statistically elevated in patients with CRSwNP than in CRSsNP and in control subjects. Besides, IL-20R2 and MMP-9 mRNA expression was increased in patients with CRSsNP than in control subjects (Fig. 1a). Immunofluorescence double staining was used to further evaluate the probable interaction between IL-19 and MMP-9. IL-19 was mainly found colocalized with MMP-9 in the epithelial cells of CRS mucosa (Fig. 1b). The above results suggested functional correlation of IL-19 with MMP-9 in the mucosal tissue of patients with CRS.

\section{2 | IL-19 induced MMP-9 expression in HNECs}

Considering the co-localization of IL-19 and MMP-9 in the mucosa of patients with CRS, we next explored whether IL-19 could elevate MMP-9 secretion in HNECs. HNECs were treated with IL-19 $(0-200 \mathrm{ng} / \mathrm{mL})$ for $24 \mathrm{~h}$. Thereafter, RT-qPCR was used to assess mRNA levels while ELISA, western blotting, and immunofluorescence were applied to evaluate protein expression. As expected, both mRNA and protein 
expression of MMP-9 were statistically elevated in response to IL-19 (Fig. 2a-d); the optimal concentration of IL-19 was $100 \mathrm{ng} / \mathrm{mL}$.

\section{3 | IL-19 promoted MMP-9 expression via NF-kB pathway in HNECs}

The MMP-9 gene promoter includes several transcription factors, and NF-kB was verified as a binding site.[25] To confirm the signaling pathways that induce up-regulated MMP-9 production in HNECs in response to IL-19, first we evaluated whether IL-19 could promote the activation of NF-kB in HNECs. HNECs were treated with IL-19 at optimal concentrations (100 ng/mL), and phosphorylation level of IKBa protein was measured by western blot while P65 nuclear translocation was detected by confocal microscopy. Results suggested treatment with IL-19 to elevated the phosphorylation of IKBa effectively in HNECs (Fig. 3a). Besides, compared to untreated HNECs, the DNA-binding capacity of NF-kB factor p65 was increased in response to IL-19 treatment (Fig. 3b). To further determine whether IL-19 upregulated MMP-9 by activating NF-kB pathway, HNECs were pre-incubated with BAY 11-7082 (IKBa-specific inhibitor) for $1 \mathrm{~h}$ before IL-19 stimulation. When BAY 11-7082 was used, both MMP-9 mRNA and protein, promoted by IL-19, were attenuated significantly, as seen in RT-qPCR and western blot, respectively (Fig. $3 c, d)$. Collectively, therefore, these findings confirmed NF-kB pathway to participate in IL-19-promoted MMP-9 production in HNECs.

\section{4 | IL-19 upregulated MMP-9 expression via ERK pathway in HNECs}

Since earlier studies had demonstrated the NF-kB pathway to be regulated by ERK signaling[10], we investigated the effect of IL-19 on upstream signaling molecules in MAPK signaling. HNECs were stimulated by IL-19 and collected at a time gradient ( $0-20 \mathrm{~min})$. Stimulation of HNECs with IL-19 induced an effective activation of MEK1/2 (Fig. 4a) and ERK1/2 (Fig. 4b), as seen in western blot. To determine whether the activation of ERK signaling was liable for IL-19-promoted MMP-9 production, HNECs were preincubated with PD98059 (MEK1/2 inhibitor) followed by IL-19 stimulation. However, preincubation with PD98059 attenuated the IL-19-promoted MMP-9 expression in HNECs (Fig. 4c, d), thus indicating the participation of ERK signaling in IL-19-induced MMP-9 production in HNECs.

Since the results suggested inhibition of both ERK and NF-kB to block the IL-19-induced MMP-9 production in HNECs, we next assessed whether ERK signaling included the pathways that resulted in the combined activation of NF-kB in IL-19-treated HNECs. HNECs were treated with PD98059 or BAY 11-7082 in presence of IL-19, and phosphorylation of IKBa and ERK1/2 was checked by western blotting. Results indicated pretreatment of HNECs with PD98059 to attenuate IL-19-promoted phosphorylation of IKBa while preincubation with BAY 11-7082 had no effect on IL-19-promoted activation of ERK1/2 in HNECs (Fig. 4e). These findings demonstrated the participation of ERK signaling in IL-19-promoted MMP-9 production by activating NF-kB in HNECs.

\section{5 | IL-19 stimulated MMP-9 production by binding to its receptor in HNECs}


IL-19 transmits its signal by binding to its receptors (IL-20R1/IL-20R2 receptor)[26]. To determine whether IL-19 stimulated MMP-9 secretion through IL-20R1 receptor, HNECs were pretreated with IL-20R1 siRNA for $48 \mathrm{~h}$ before being stimulated by IL-19. We found the interference of IL-20R1 to significantly attenuate the activation of ERK1/2 and IKBa in HNECs in response to IL-19 (Fig. 5a, b). Besides, si-IL-20R1 significantly inhibited the IL-19-promoted MMP-9 expression in HNECs, as seen by RT-qPCR (Fig. 5c), western blotting (Fig. 5d), and immunofluorescence (Fig. 5e). These findings together demonstrated IL-19 to possibly mediate ERK and NF-kB pathway activation, and MMP-9 production in HNECs by binding to IL-20R1.

\section{6 | IL-13 and IL-17A upregulated IL-19 production in HNECs}

To determinate whether the expression of IL-19 was regulated by cytokines, HNECs were treated with type 1 cytokines (IFN-y, IL-1 $\beta$ ), type 2 cytokines (IL-4, IL-5 and IL-13), type 3 cytokines (IL-17A), and IL-25. RNA was collected, after 6-h stimulation, for conducting RT-qPCR, and proteins were extracted after 24-h treatment for western blot. Only IL-13 and IL-17A were found to promote IL-19 expression in RT-qPCR (Fig. 6a) and western blot (Fig. 6b). In order to confirm whether IL-13 and IL-17A could elevate MMP-9 secretion bypass IL-19 production, HNECs were pretreated with IL-20R1 siRNA for $48 \mathrm{~h}$ before stimulation with IL-13 and IL-17A. The protein levels of MMP-9 were effectively increased in response to IL-13 and IL17A. Moreover, the promoting effect of IL-13 and IL-17A on MMP-9 production could be partly suppressed by IL-20R1 siRNA (Fig. 6c, d). Collectively, our data showed IL-13 and IL-17A cytokines to be capable of promoting the secretion of IL-19 in HNECs.

\section{4 | Discussion}

One of the main events in tissue remodeling is the degradation of ECM components by proteolytic enzymes.[27] Insufficient suppression of MMP-9 by TIMP-1 in polyp could lead to degradation of ECM, and hence, pseudocyst formation.[28] However, TGF- $\beta 1$ can promote TIMP-1 expression, and elevated levels of TGF- $\beta 1$ and TIMP-1, relative to MMP-9, may account for the principal fibrosis observed in CRSsNP.[29] In our previous study[12] and the current one, we demonstrated MMP-7 and MMP-9 expression in CRSwNP to be elevated compared to that in CRSsNP and the control group, which was consistent with other previous reports.[11, 14, 29] MMPs could be an important treatment target for chronic rhinosinusitis. As one of the MMPs inhibitors, doxycycline has been demonstrated to effectually diminish MMP-9 expression in nasal secretions, eventually shrinking the size of polyps.[30] Studies have also shown high concentrations of MMP-9 after functional endoscopic sinus surgery (FESS) to be related to bad treatment outcome.[31] Doxycycline-releasing sinus stents have been shown to effectively reduce nasal MMP-9 concentrations and improve therapeutic effect in patients post FESS.[32] Therefore, the upstream regulators of MMP-9 in CRS would be worth studying.

Recent reports have indicated IL-20 subfamily to participate in many inflammatory diseases, liver fibrosis, rheumatoid arthritis, psoriasis, and asthma.[33-36] Our previous studies had shown IL-19 to be involved in mucin production, a participant in tissue remodeling in CRS.[37] In the present study, mRNA levels of IL- 
19, its receptors (IL-20R1/IL-20R2), and MMP-9 were found elevated in CRSwNP. Additionally, IL-19 was mainly seen to be co-localized with MMP-9 in the epithelium of CRS mucosa. According to the above results, we speculated IL-19 to possibly participate in the development and progression of tissue remodeling in CRS. Thereafter, we aimed to investigate how IL-19 could conduce to the modulation of MMP-9 production in HNECs. As expected, IL-19 stimulation was observed to promote MMP-9 production in HNECs at an optimal concentration of $100 \mathrm{ng} / \mathrm{mL}$. Additionally, transfection of IL-20R1 siRNA into HNECs effectively attenuated IL-19-promoted MMP-9 production. The function of IL-19 might manifest after combining with IL-20R1 receptor, thus resulting in MMP-9 overexpression and ECM destruction. This finding was in line with studies concerning the function of IL-19 depending on its binding to a functional heterodimeric receptor IL-20R1/IL-20R2.[26] In contrast, IL-19 had no effect on the expression of MMP-7 in HNECs (Supplement Fig. 1).

Till date, only a few studies have been reported about the specific mechanism of MMP-9 modulation in CRS. Previous studies had demonstrated IL-20 subfamily members to activate the signal transducer and activator of transcription (STAT), Janus Kinase (JAK), and mitogen-activated protein kinase (MAPK) signaling pathways.[15] As a subclass of MAPK pathways, the ERK signaling pathway is also activated by a sequence of phosphorylation events in response to extracellular stimuli, which then transmits extracellular signals that induce cellular proliferation, differentiation, and survival. After being activated, RAF kinases (MAPKKK) phosphorylate and activate the elements of MAPKK module MEK1/2, which then activate the MAPK protein kinase, ERK1/2. Once activated, ERK1/2 phosphorylates and connects various extracellular signals to cytoplasm or nucleus.[38] In our current study, we demonstrated effective induction of phosphorylation of MEK1/2 and ERK1/2, modules of ERK pathway, and expression of MMP9 in HNECs by IL-19. We also identified the participation of transcription factor NF-kB in ERK-regulated MMP-9 production in IL-19-stimulated HNECs. These findings were similar to that reported in studies concerning atherosclerosis and bladder cancer. In reports regarding the pathogenesis of atherosclerosis, CD147 had been demonstrated to mediate MMP-9 induction through ERK and the nuclear translocation of NF-kB pathway in macrophage[39]. IL-20 could also induce bladder cancer cell migration by activating the ERK-mediated NF-kB pathway, and promoting MMP-9 production subsequently.[18] Regulatory components that exist in the MMP-9 gene contain the binding site for NF-kB, which has been proved to drive MMP-9 production in macrophages, neutrophils, cardiomyocytes, fibroblasts, and bladder cancer cells.[18, 25, 40-42] As a dimeric transcription factor (p50/p65), the classical NF-kB acts as a pivotal inflammatory regulator and modulates various genes. Upon activation, NF-kB translocates to the nucleus, after which the active subunit p65 promotes transcription of chemokines, cytokines, and adhesion molecules.[43] We demonstrated the promotion of NF-kB activation by IL-19, which generated phosphorylation of IKBa and translocation of p65 subunit, accompanied by increased MMP-9 expression in HNECs. Besides, this excessive expression of MMP-9 could be eliminated by NF-kB pathway-specific inhibitor BAY 11-7082. Furthermore, our study on the inhibitor PD98059 suggested the classical NF-kB pathway, activated by IL-19, to be mediated by ERK signaling.

Accumulating studies have shown inflammatory cytokines to be important components of the nasal environment of CRS, and to participate in tissue remodeling.[44] Although macrophages, keratinocytes, 
fibroblasts, airway epithelial cells, and nasal epithelia have been demonstrated to be the cellular sources of IL-19[15, 19], very few studies have focused on the stimulatory effects of cytokines on IL-19 production, especially in CRS. However, IL-4, IL-13, and IL-17A have been proven to perform stimulatory function in IL-19 production in airway epithelia. Besides, IL-13 and IL-17A collectively act to further stimulate IL-19 production through a STAT6-dependent transcriptional pathway in airway epithelial cells. [45] Similar to this study, we observed IL-13 and IL-17A to be capable of up-regulating IL-19 production in HNECs. Furthermore, the acceleration of MMP-9 production by IL-13 and IL-17A could be partly suppressed by IL-20R1 siRNA. Our study suggested IL-13 and IL-17A to possibly participate in the tissue remodeling of CRS by positively regulating the MMP-9 expression through IL-19 secretion in HNECs. It indicated that CRSwNP subgroups with different clinical characteristics might eventually cause similar tissue remodeling patterns by affecting IL-19 expression. Our findings provide important insights into the pathogenesis of CRS and propose a potential therapeutic target to suppress pathological nasal tissue remodeling in future.

In conclusion, we demonstrated prominent expression of IL-19 in nasal polyps of patients with CRSwNP, along with increased expression of tissue remodeling factor MMP-9. We then focused on the role of IL-19 in modulating MMP-9 expression in HNECs. Based on our findings, we proposed the novel concept of IL19 up-regulating MMP-9 production by binding to IL-20R1 in HNECs. Moreover, IL-19 promoted MMP-9 expression by stimulating the classical NF-kB pathway, mediated by ERK signaling. Our results also suggested IL-13 and IL-17A to elevate IL-19 expression in HNECs. Collectively, our findings illustrated the participation of IL-19 (promoted by IL-13 and IL-17A) in tissue remodeling in patients with CRS.

\section{Declarations}

\section{ACKNOWLEDGMENTS}

This work was supported by the National Natural Science Foundation of China (grant numbers 81670913, 81970859), the Natural Science Foundation of Guangdong Province (grant number 2019A1515011093), and the Young Teacher Foundation of Sun Yat-sen University (grant number 19ykpy30).

\section{CONFLICTS OF INTEREST}

The authors declare that they have no conflicts of interest.

\section{AUTHOR CONTRIBUTIONS}

$\mathrm{Li}$, J.-C. Huang, and X.-H. Chen performed RT-qPCR, western blot, and ELISA, and prepared the manuscript; X.-P. Lai and Z.-Z.H performed IF; Y. Li and S.-X. Li participated in sample collection; G.-H. Zhang and L.-H. Chang designed the study and prepared the manuscript.

\section{References}


1. Hastan D, Fokkens WJ, Bachert C, Newson RB, Bislimovska J, Bockelbrink A, et al. Chronic rhinosinusitis in Europe--an underestimated disease. A GA²LEN study. Allergy. 2011;66(9):1216-23.

2. Blackwell DL, Lucas JW, Clarke TC. Summary health statistics for U.S. adults: national health interview survey, 2012. Vital Health Stat 10. 2014;(260):1-161.

3. Shi JB, Fu QL, Zhang H, Cheng L, Wang YJ, Zhu DD, et al. Epidemiology of chronic rhinosinusitis: results from a cross-sectional survey in seven Chinese cities. Allergy. 2015;70(5):533-9.

4. Bhattacharyya N, Orlandi RR, Grebner J, Martinson M. Cost burden of chronic rhinosinusitis: a claims-based study. Otolaryngol Head Neck Surg. 2011;144(3):440-5.

5. Stevens WW, Lee RJ, Schleimer RP, Cohen NA. Chronic rhinosinusitis pathogenesis. J Allergy Clin Immunol. 2015;136(6):1442-53.

6. Liao B, Liu JX, Li ZY, Zhen Z, Cao PP, Yao Y, et al. Multidimensional endotypes of chronic rhinosinusitis and their association with treatment outcomes. Allergy. 2018;73(7):1459-69.

7. Tomassen P, Vandeplas G, Van Zele T, Cardell LO, Arebro J, Olze H, et al. Inflammatory endotypes of chronic rhinosinusitis based on cluster analysis of biomarkers. J Allergy Clin Immunol. 2016;137(5):1449-56.e4.

8. Yang YC, Zhang N, Van Crombruggen K, Hu GH, Hong SL, Bachert C. Transforming growth factorbeta 1 in inflammatory airway disease: a key for understanding inflammation and remodeling. Allergy. 2012;67(10):1193-202.

9. Kato A. Immunopathology of chronic rhinosinusitis. Allergol Int. 2015;64(2):121-30.

10. Chen Q, Jin M, Yang F, Zhu J, Xiao Q, Zhang L. Matrix metalloproteinases: inflammatory regulators of cell behaviors in vascular formation and remodeling. Mediators Inflamm. 2013;2013:928315.

11. Li X, Meng J, Qiao X, Liu Y, Liu F, Zhang N, et al. Expression of TGF, matrix metalloproteinases, and tissue inhibitors in Chinese chronic rhinosinusitis. J Allergy Clin Immunol. 2010;125(5):1061-8.

12. Yang LY, Li X, Li WT, Huang JC, Wang ZY, Huang ZZ, et al. VY1区 Y $\delta T$ Cells Are Correlated With Increasing Expression of Eosinophil Cationic Protein and Metalloproteinase-7 in Chronic Rhinosinusitis With Nasal Polyps Inducing the Formation of Edema. Allergy Asthma Immunol Res. 2017;9(2):142-51.

13. Chen X, Chang L, Li X, Huang J, Yang L, Lai X, et al. Tc17/IL-17A Up-Regulated the Expression of MMP-9 via NF-KB Pathway in Nasal Epithelial Cells of Patients With Chronic Rhinosinusitis. Front Immunol. 2018;9:2121.

14. Kim DK, Eun KM, Kim MK, Cho D, Han SA, Han SY, et al. Comparison Between Signature Cytokines of Nasal Tissues in Subtypes of Chronic Rhinosinusitis. Allergy Asthma Immunol Res. 2019;11(2):20111.

15. Rutz S, Wang X, Ouyang W. The IL-20 subfamily of cytokines-from host defence to tissue homeostasis. Nat Rev Immunol. 2014;14(12):783-95.

16. Autieri MV. IL-19 and Other IL-20 Family Member Cytokines in Vascular Inflammatory Diseases. Front Immunol. 2018;9:700. 
17. Huang KY, Lin RM, Chen WY, Lee CL, Yan JJ, Chang MS. IL-20 may contribute to the pathogenesis of human intervertebral disc herniation. Spine (Phila Pa 1976). 2008;33(19):2034-40.

18. Lee SJ, Cho SC, Lee EJ, Kim S, Lee SB, Lim JH, et al. Interleukin-20 promotes migration of bladder cancer cells through extracellular signal-regulated kinase (ERK)-mediated MMP-9 protein expression leading to nuclear factor (NF-KB) activation by inducing the up-regulation of p21(WAF1) protein expression. J Biol Chem. 2013;288(8):5539-52.

19. Pace E, Scafidi V, Di Bona D, Siena L, Chiappara G, Ferraro M, et al. Increased expression of IL-19 in the epithelium of patients with chronic rhinosinusitis and nasal polyps. Allergy. 2012;67(7):878-86.

20. Watelet JB, Dogne JM, Mullier F. Remodeling and Repair in Rhinosinusitis. Curr Allergy Asthma Rep. 2015;15(6):34.

21. Cao PP, Li HB, Wang BF, Wang SB, You XJ, Cui YH, et al. Distinct immunopathologic characteristics of various types of chronic rhinosinusitis in adult Chinese. J Allergy Clin Immunol. 2009;124(3):478-84, 484.e1-2.

22. Cao PP, Wang ZC, Schleimer RP, Liu Z. Pathophysiologic mechanisms of chronic rhinosinusitis and their roles in emerging disease endotypes. Ann Allergy Asthma Immunol. 2019;122(1):33-40.

23. Fokkens WJ, Lund VJ, Mullol J, Bachert C, Alobid I, Baroody F, et al. EPOS 2012: European position paper on rhinosinusitis and nasal polyps 2012. A summary for otorhinolaryngologists. Rhinology. 2012;50(1):1-12.

24. Li X, Wang Z, Chang L, Chen X, Yang L, Lai X, et al. үठT cells contribute to type 2 inflammatory profiles in eosinophilic chronic rhinosinusitis with nasal polyps. Clin Sci (Lond). 2019;133(22):230115.

25. lyer RP, Jung M, Lindsey ML. MMP-9 signaling in the left ventricle following myocardial infarction. Am J Physiol Heart Circ Physiol. 2016;311(1):H190-8.

26. Sabat R, Wallace E, Endesfelder S, Wolk K. IL-19 and IL-20: two novel cytokines with importance in inflammatory diseases. Expert Opin Ther Targets. 2007;11(5):601-12.

27. Van Bruaene $\mathrm{N}$, Bachert $\mathrm{C}$. Tissue remodeling in chronic rhinosinusitis. Curr Opin Allergy Clin Immunol. 2011;11(1):8-11.

28. Lee YM, Kim SS, Kim HA, Suh YJ, Lee SK, Nahm DH, et al. Eosinophil inflammation of nasal polyp tissue: relationships with matrix metalloproteinases, tissue inhibitor of metalloproteinase-1, and transforming growth factor-beta1. J Korean Med Sci. 2003;18(1):97-102.

29. Watelet JB, Bachert C, Claeys C, Van Cauwenberge P. Matrix metalloproteinases MMP-7, MMP-9 and their tissue inhibitor TIMP-1: expression in chronic sinusitis vs nasal polyposis. Allergy. 2004;59(1):54-60.

30. Van Zele T, Gevaert P, Holtappels G, Beule A, Wormald PJ, Mayr S, et al. Oral steroids and doxycycline: two different approaches to treat nasal polyps. J Allergy Clin Immunol. 2010;125(5):1069-76.e4.

31. Watelet JB, Demetter P, Claeys C, Van Cauwenberge P, Cuvelier C, Bachert C. Neutrophil-derived metalloproteinase-9 predicts healing quality after sinus surgery. Laryngoscope. 2005;115(1):56-61. 
32. Huvenne W, Zhang N, Tijsma E, Hissong B, Huurdeman J, Holtappels G, et al. Pilot study using doxycycline-releasing stents to ameliorate postoperative healing quality after sinus surgery. Wound Repair Regen. 2008;16(6):757-67.

33. Liao SC, Cheng YC, Wang YC, Wang CW, Yang SM, Yu CK, et al. IL-19 induced Th2 cytokines and was up-regulated in asthma patients. J Immunol. 2004;173(11):6712-8.

34. Chiu YS, Wei CC, Lin YJ, Hsu YH, Chang MS. IL-20 and IL-20R1 antibodies protect against liver fibrosis. Hepatology. 2014;60(3):1003-14.

35. Kragstrup TW, Andersen T, Heftdal LD, Hvid M, Gerwien J, Sivakumar P, et al. The IL-20 Cytokine Family in Rheumatoid Arthritis and Spondyloarthritis. Front Immunol. 2018;9:2226.

36. Wei CC, Chen WY, Wang YC, Chen PJ, Lee JY, Wong TW, et al. Detection of IL-20 and its receptors on psoriatic skin. Clin Immunol. 2005;117(1):65-72.

37. Lai X, Li X, Chang L, Chen X, Huang Z, Bao H, et al. IL-19 Up-Regulates Mucin 5AC Production in Patients With Chronic Rhinosinusitis via STAT3 Pathway. Front Immunol. 2019;10:1682.

38. Mao LM, Wang JQ. Synaptically Localized Mitogen-Activated Protein Kinases: Local Substrates and Regulation. Mol Neurobiol. 2016;53(9):6309-15.

39. Kim JY, Kim WJ, Kim H, Suk K, Lee WH. The Stimulation of CD147 Induces MMP-9 Expression through ERK and NF-kappaB in Macrophages: Implication for Atherosclerosis. Immune Netw. 2009;9(3):90-7.

40. McDonald PP, Bald A, Cassatella MA. Activation of the NF-kappaB pathway by inflammatory stimuli in human neutrophils. Blood. 1997;89(9):3421-33.

41. Thorp EB. Contrasting Inflammation Resolution during Atherosclerosis and Post Myocardial Infarction at the Level of Monocyte/Macrophage Phagocytic Clearance. Front Immunol. 2012;3:39.

42. Van Linthout S, Miteva K, Tschöpe C. Crosstalk between fibroblasts and inflammatory cells. Cardiovasc Res. 2014;102(2):258-69.

43. Liu T, Zhang L, Joo D, Sun SC. NF-KB signaling in inflammation. Signal Transduct Target Ther. 2017;2.

44. Van Crombruggen K, Zhang N, Gevaert P, Tomassen P, Bachert C. Pathogenesis of chronic rhinosinusitis: inflammation. J Allergy Clin Immunol. 2011;128(4):728-32.

45. Huang F, Wachi S, Thai P, Loukoianov A, Tan KH, Forteza RM, et al. Potentiation of IL-19 expression in airway epithelia by IL-17A and IL-4/IL-13: important implications in asthma. J Allergy Clin Immunol. 2008;121(6):1415-21, 1421.e1-3.

\section{Figures}


A

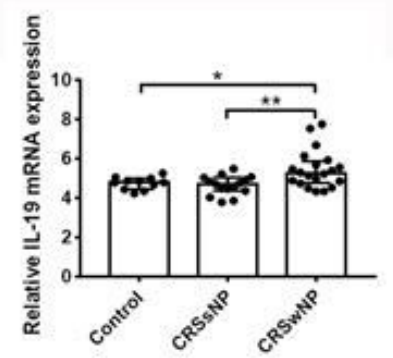

IL-19

B
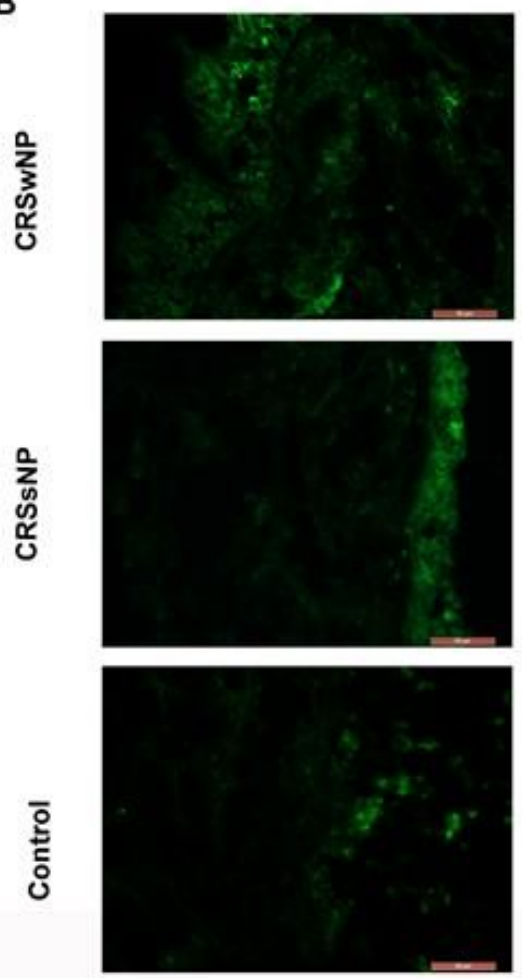

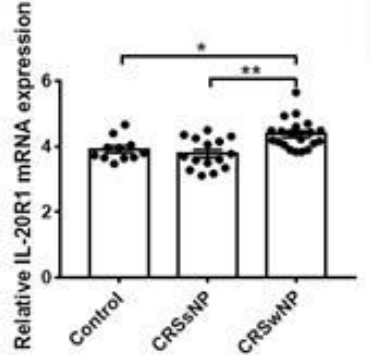

MMP-9
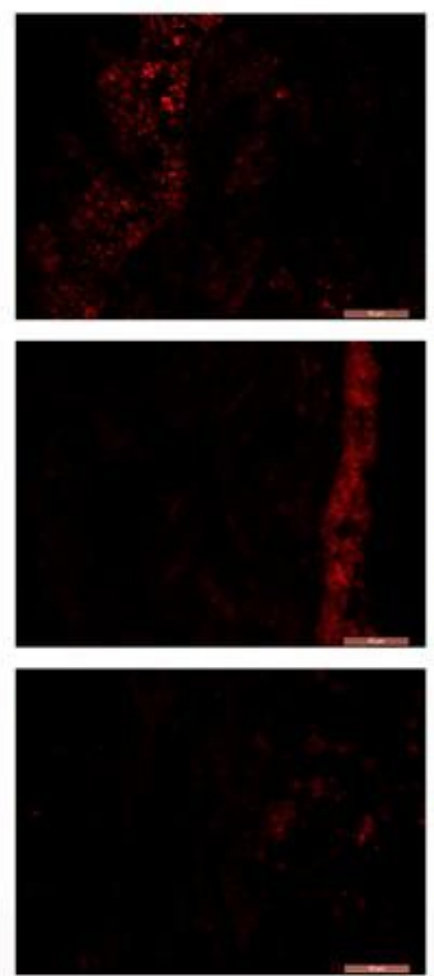

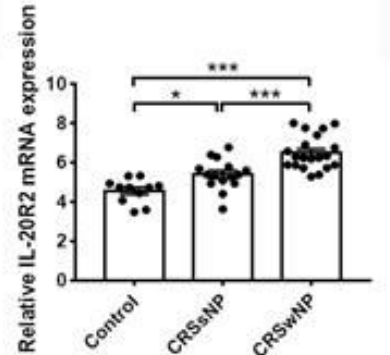

DAPI
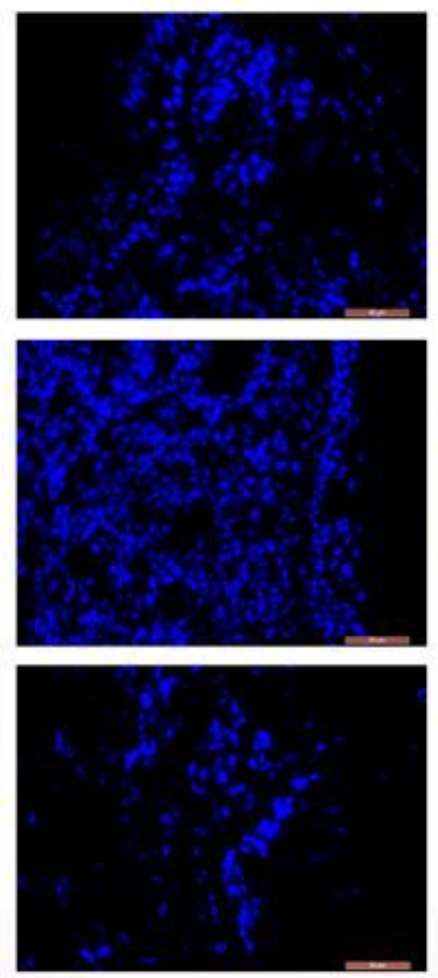

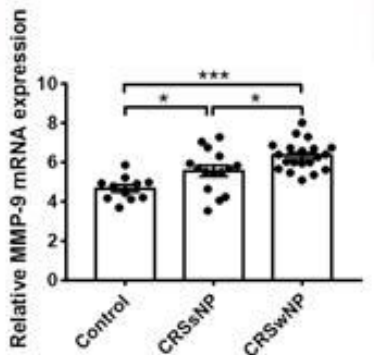

Merge
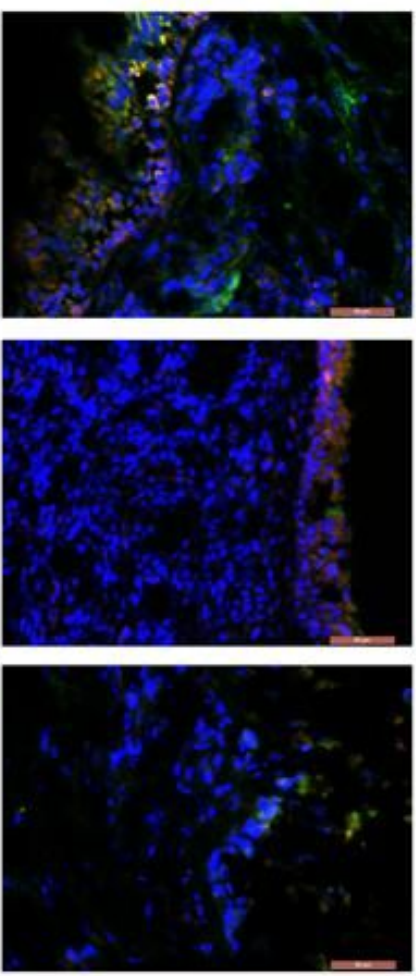

\section{Figure 1}

Increased expression of IL-19, its receptors (IL-20R1/IL-20R2), and MMP-9 in nasal mucosa of patients with CRSwNP a RT-qPCR results; relative mRNA levels of IL-19, its receptors (IL-20R1/IL-20R2), and MMP9 (CRSwNP 20, CRSsNP 15, Control 10, and IL-19 are expressed as median (IQRs); IL-20R1, IL-20R2, and MMP-9 are expressed as mean \pm SEM); b Nuclei, IL-19, and MMP-9 are stained in blue, green, and red by immunofluorescence, respectively (magnification, 400x) $* \mathrm{P}<0.05$, ** $\mathrm{P}<0.01$, $* \star * P<0.001$. 
A

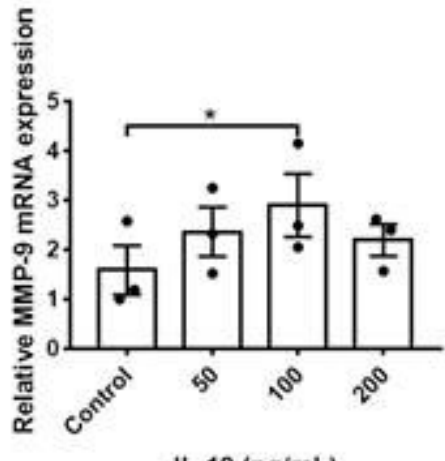

IL-19 (ng/mL)

C

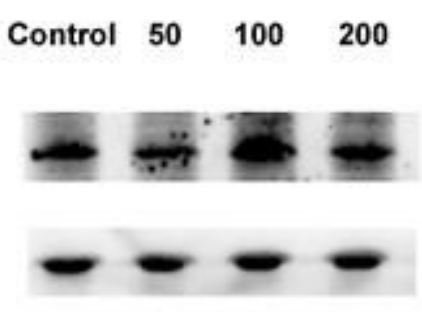

D

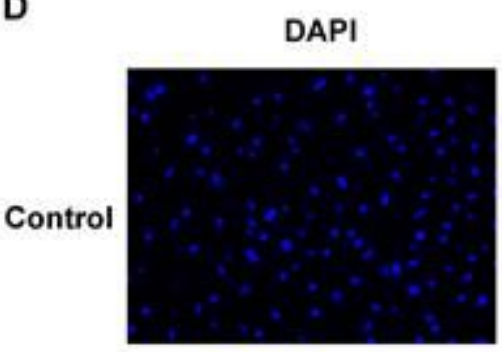

IL-19

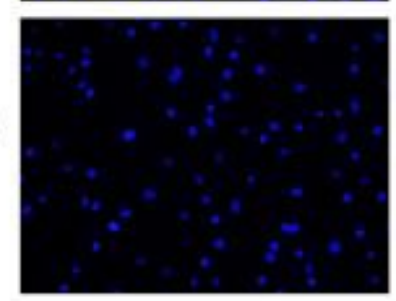

B

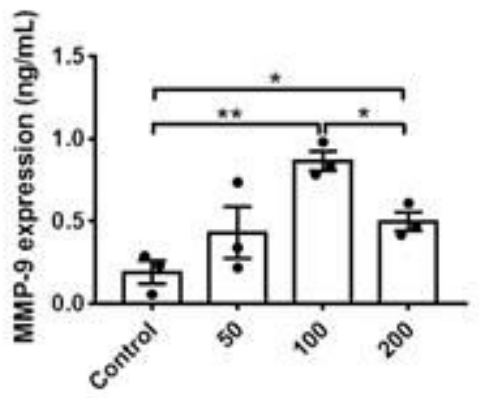

IL-19 (ng/mL)

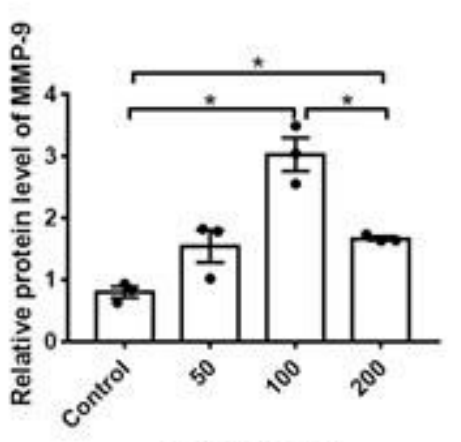

IL-19 (ng/mL)

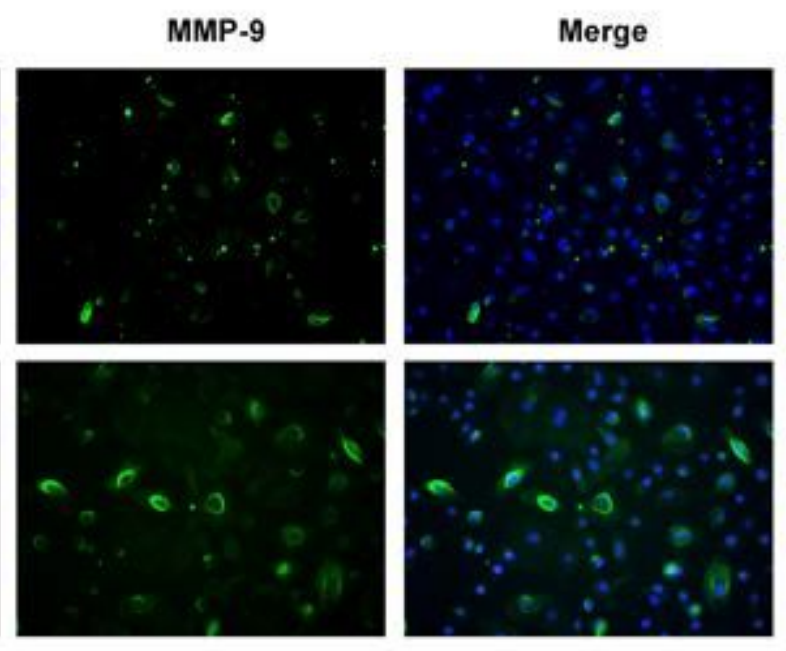

Figure 2

IL-19 induced MMP-9 expression in HNECs HNECs were incubated with gradient concentrations of IL-19 for 24 h. a, c HNECs were collected to measure transcription and protein levels of MMP-9 $(n=3)$. b HNEC supernatants were collected to measure MMP-9 release by ELISA $(n=3)$. d HNECs were collected for immunofluorescence staining to detect MMP-9 protein. ${ }^{*}<0.05$, ${ }^{\star *} \mathrm{P}<0.01$, mean $\pm \mathrm{SEM}$. 
A

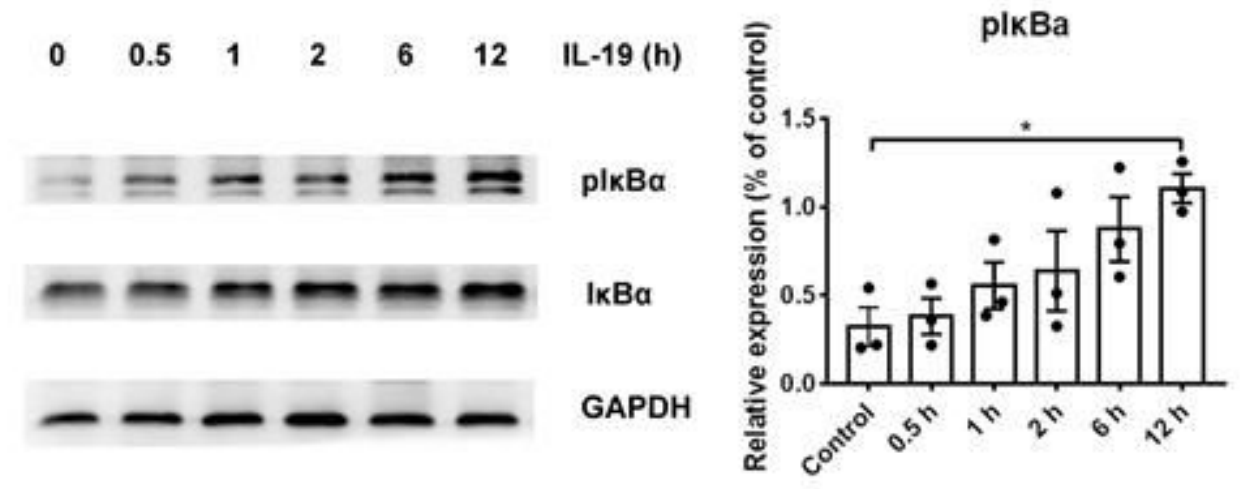

B

DAPI

p65

Merge

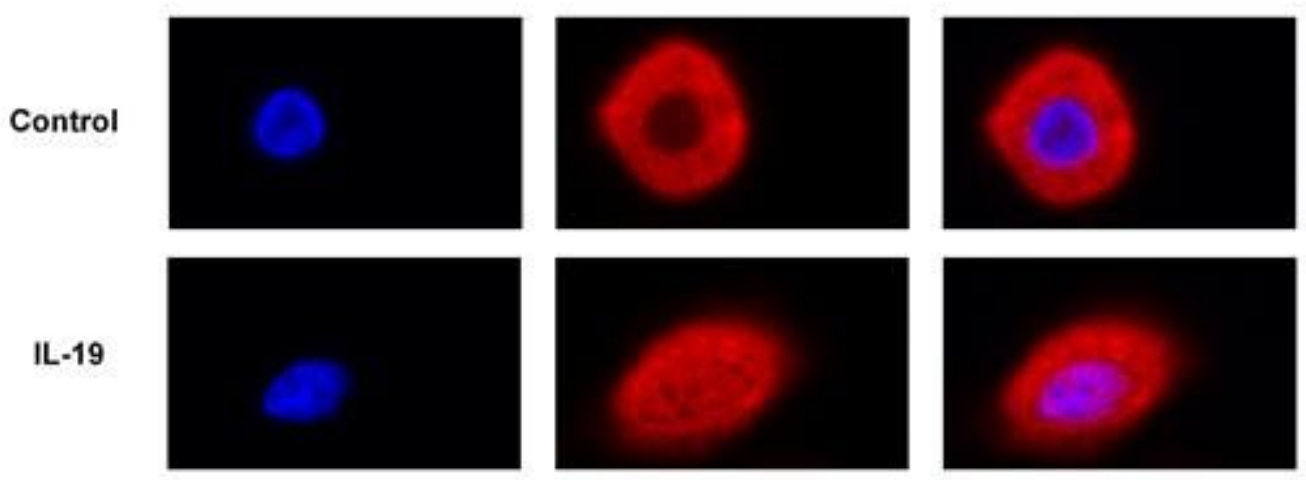

C

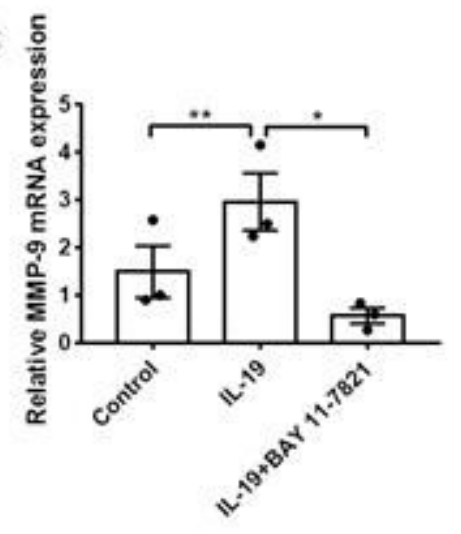

D

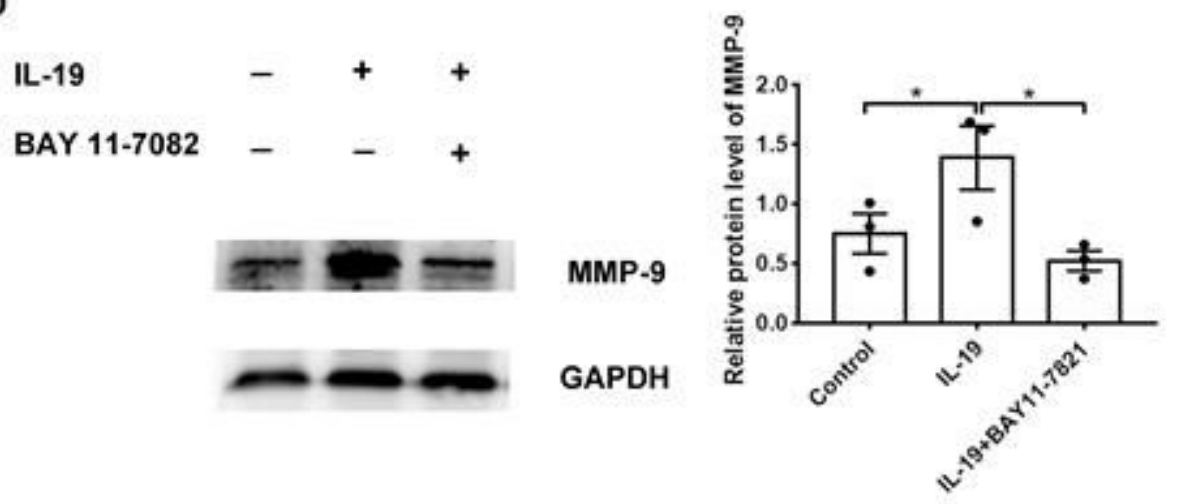

Figure 3

IL-19 promoted MMP-9 expression via NF-KB pathway in HNECs HNECs were treated with IL-19 and collected at gradient time ( $0-12 \mathrm{~h})$. a Western blotting detected the phosphorylation levels of $\mathrm{IKBa}(\mathrm{n}=3)$. b HNECs were incubated with IL-19 for $24 \mathrm{~h}$, p65 nuclear translocation was detected by confocal microscopic analysis. c, d HNECs were pretreated with BAY 11-7082 $(5 \mu \mathrm{M})$ for $1 \mathrm{~h}$ and subsequently treated with IL-19. HNECs were collected to measure transcription and protein levels of MMP-9 by RTqPCR and western blot, respectively $(n=3) .{ }^{*} P<0.05$, ${ }^{\star *} P<0.01$, mean \pm SEM. 

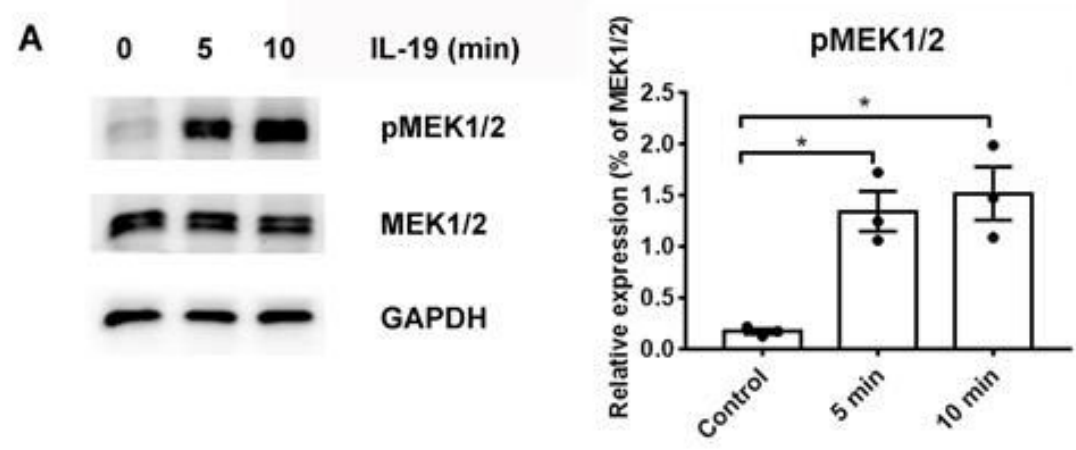

E

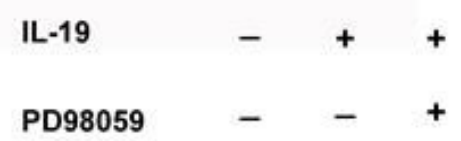

B
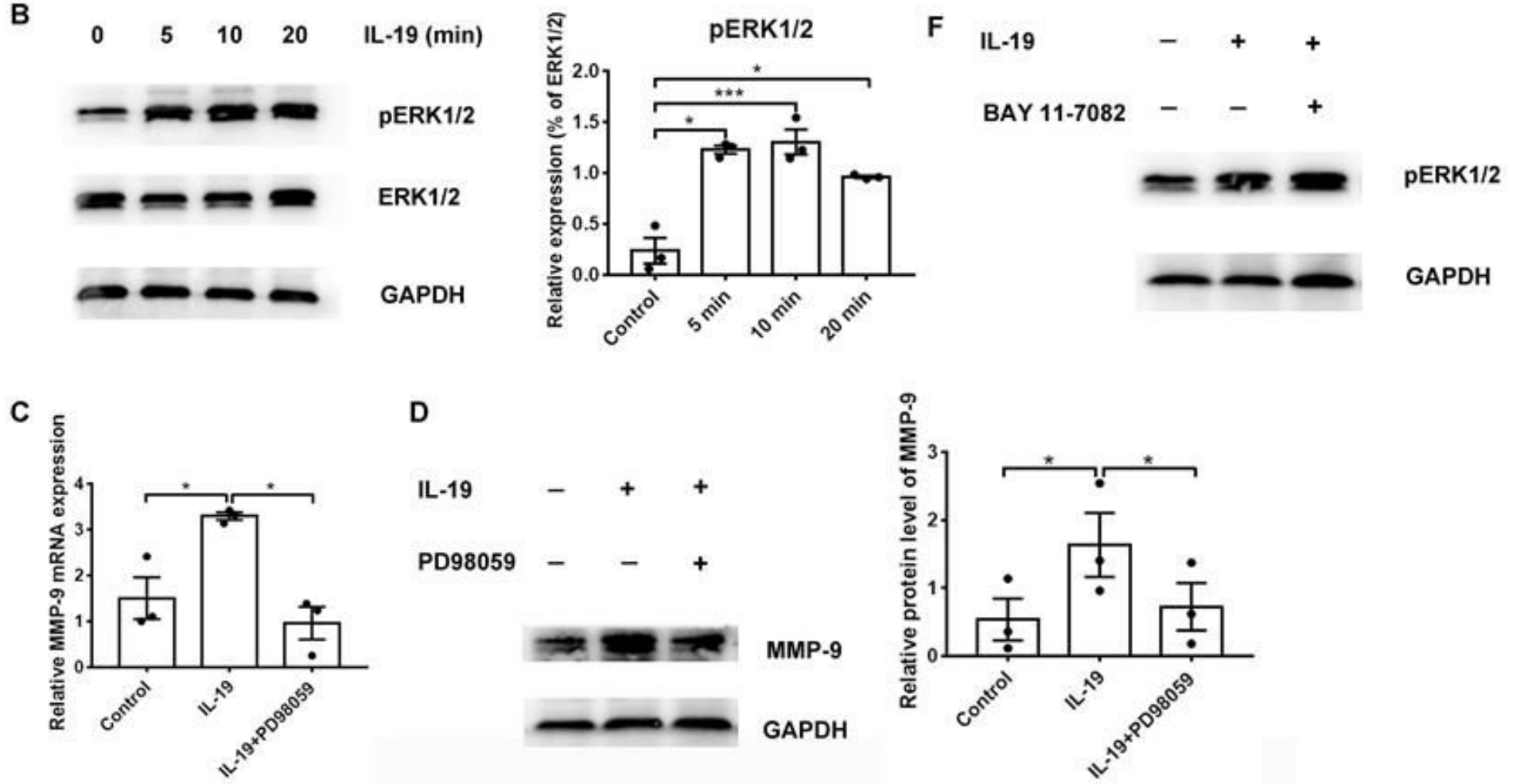

Figure 4

IL-19 upregulated MMP-9 expression via ERK pathway in HNECs a, b HNECs were treated with IL-19 and collected at gradient time (0-20 min). Thereafter, western blotting was performed to measure the phosphorylation of MEK1/2 and ERK1/2 ( $n=3)$. c, d HNECs were preincubated with PD98059 (20 $\mu$ M) for $1 \mathrm{~h}$ and subsequently treated with IL-19. They were subsequently collected to measure transcription ( $\mathrm{n}=$ 3) and protein $(n=3)$ levels of MMP-9 by RT-qPCR and western blot, respectively. e, f HNECs were pretreated with PD98059 $(20 \mu \mathrm{M})$ or BAY 11-7082 $(5 \mu \mathrm{M})$ for $1 \mathrm{~h}$ before being stimulated with IL-19, and western blotting was performed to measure the phosphorylation of IKBaor ERK $1 / 2$, respectively. ${ }^{*} P<0.05$, $\star \star P<0.01, * \star * P<0.001$, mean \pm SEM. 

A $1 \mathrm{~L}-19$

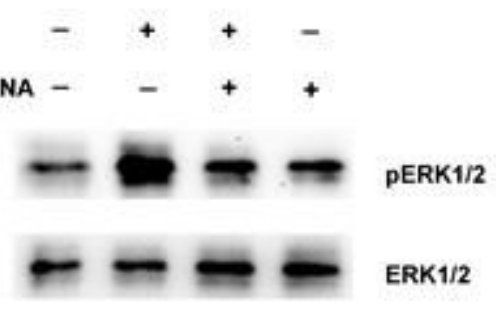
B $\mathrm{IL}-19$
IL-20R1 SiRNA -
IL-20R1 siRNA - - + -
$=0$ GAPDH

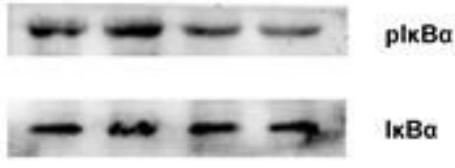
GAPDH

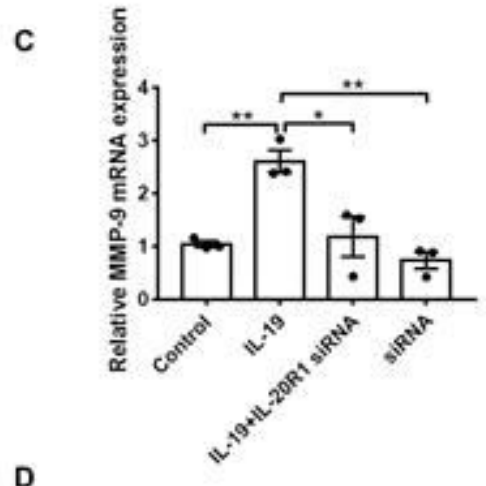

D
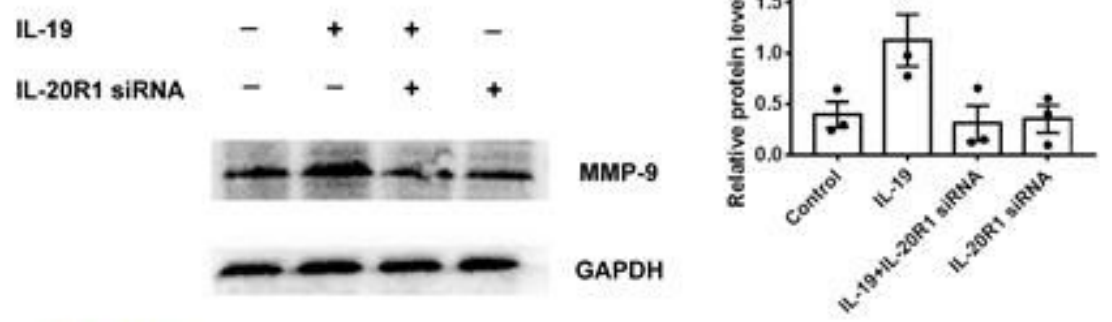

$\mathbf{E}$

MMP-9/DAPI

Control

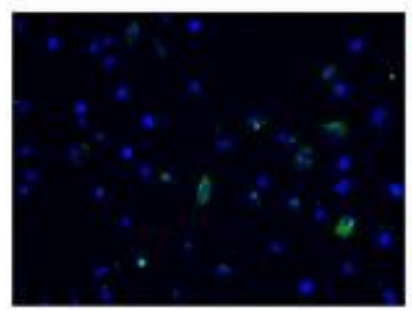

IL-19

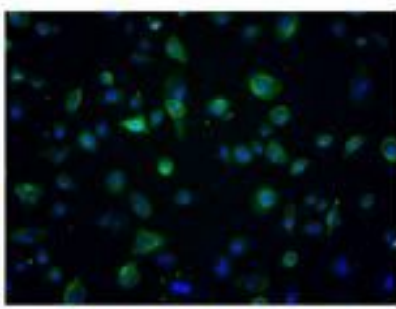

IL-19+IL-20R1 SIRNA

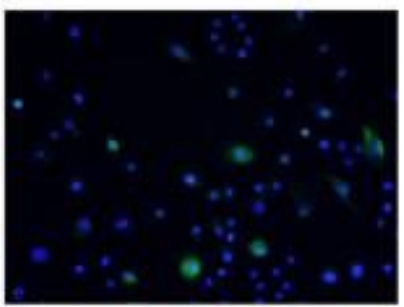

\section{Figure 5}

IL-19 stimulated MMP-9 production by binding to receptor in HNECs a, b HNECs were transfected with IL20R1 siRNA for $48 \mathrm{~h}$ and subsequently incubated with IL-19 for $24 \mathrm{~h}$. They were then collected and western blotting performed to measure the phosphorylation of ERK1/2 $(n=3)$ and IKBa $(n=3)$. c-e MMP9 expression was measured by RT-qPCR $(n=3)$, western blot $(n=3)$, and immunofluorescence. ${ }^{*}<<0.05$, $\star * P<0.01, * \star * P<0.001$, mean \pm SEM. 
A

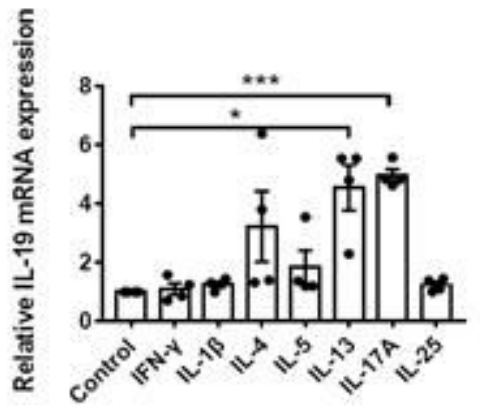

B Control IFN-y IL-1 $\beta$ IL-4 IL-5 IL-13 IL-17A IL-25

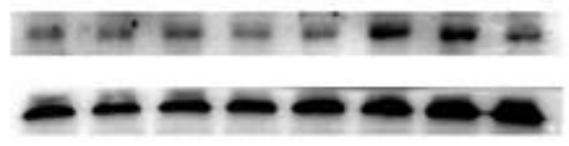

IL-19

GAPDH

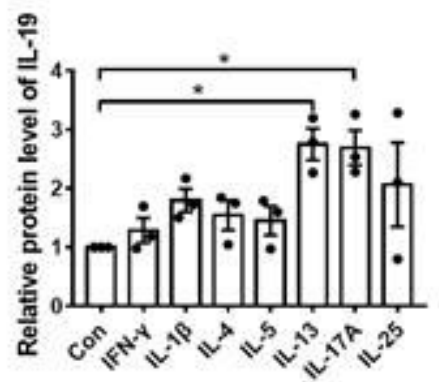

C

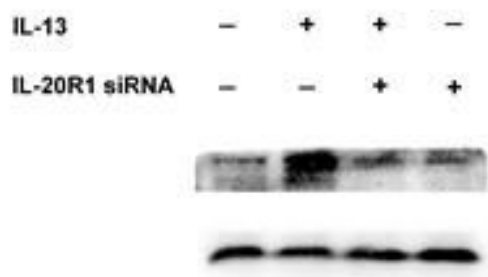

MMP.9

GAPDH

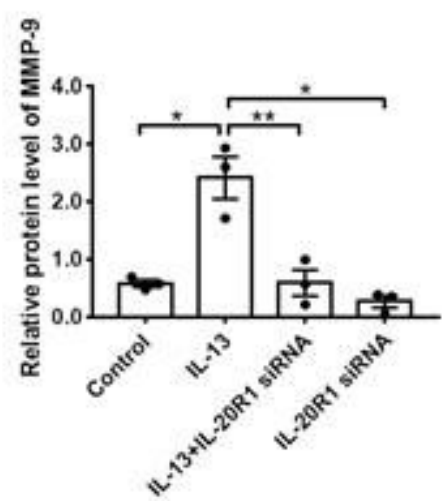

D

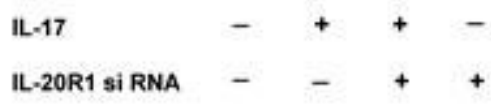

$\square=-\square$ MMP.9

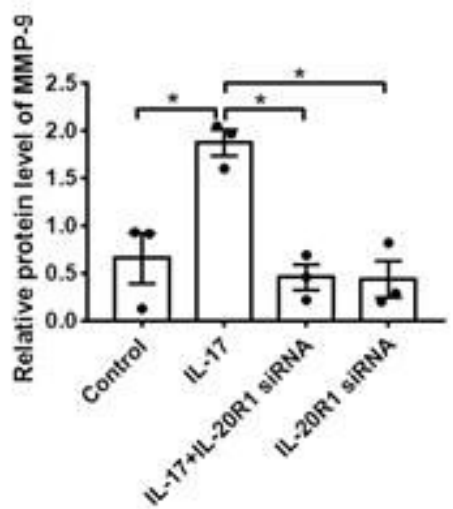

\section{Figure 6}

IL-13 and IL-17A stimulated IL-19 production in HNECs HNECs were incubated with type 1 cytokines (IFNY, IL- $\beta$ ), type 2 cytokines (IL-4, IL-5, and IL-13), type 3 cytokines (IL-17A), and IL-25 at a concentration of 20 $\mathrm{ng} / \mathrm{mL}$. a After 6-h incubation, HNECs were collected to measure transcription levels of MMP-9 $(n=4)$. $b$ After 12-h stimulation, proteins were extracted and measured by western blotting $(n=3)$. c, d HNECs were transfected with IL-20R1 siRNA for $48 \mathrm{~h}$ and subsequently stimulated with IL-13 or IL-17A for $24 \mathrm{~h}$. HNECs were collected to measure MMP-9 expression, and analyzed using quantitative western blot $(n=3)$. * $<<$ $0.05, * * P<0.01, * * * P<0.001$, mean \pm SEM.

\section{Supplementary Files}


This is a list of supplementary files associated with this preprint. Click to download.

- IL19supplementarymaterials.docx 